\title{
Design of a Wideband Harmonically Tuned Class-J Power Amplifier
}

\author{
Maryam Sajedin ${ }^{1}$, Issa Elfergani ${ }^{1}$, Jonathan Rodriguez ${ }^{1}$ \\ Manuel Violas ${ }^{1}$, Raed Abd_Alhameed ${ }^{2}$, Monica Fernandez-Barciela ${ }^{3}$ \\ Ahmed M.Abdulkhaleq ${ }^{4}$ \\ \{Maryam.sajedin*, i.t.e.elfergani, jonathan @av.it.pt ${ }^{1}$ \}
}

Instituto de Telecomunicações, Aveiro, Portugal ${ }^{1}$, Faculty of Engineering and Informatics, Bradford University, Bradford BD7 1DP, UK ${ }^{2}$, Department of Signal Theory and Communications, University of Vigo, 36310 Vigo, Spain $^{3}$, SARAS Technology Limited, Leeds LS12 4NQ, UK ${ }^{4}$

\begin{abstract}
This paper describes a modern design idea of wideband and high efficient GaN HEMT Class-J power amplifiers. The operation principle of the Class-J mode, based on the second harmonic tuning, is introduced which can maximize the achievable efficiency. Matching network synthesis criteria for wideband frequency response are addressed for $5 \mathrm{G}$ power amplifiers design. This wideband harmonically tuned technique is applicable to amplifiers based on adjusting the shape of the waveforms through a a finite number of harmonics, such as Class-F, $\mathrm{F}^{-1}$ and E. Simulation results indicate that Class-J can provide a high drain efficiency of $70 \%$ across a 1.5 to $3.2 \mathrm{GHz}$ bandwidth while delivering $10 \mathrm{~W}$ output power at the $1 \mathrm{~dB}$ - compression point, using GaN technology.
\end{abstract}

Keywords: Power Amplifier design, Class-J, Broadband, High efficiency

\section{Introduction}

Development of green technology is expected to achieve significant reduction in energy usage by $90 \%$ and multimode operation to support interoperability (sub 6GHz and mmWave). Moreover, the $5 \mathrm{G}$ cellular service providers are deploying distributed antennas and advanced modulations to provide ten times higher data rate connectivity than LTE networks, that require larger spectrum allocations. Power Amplifier (PA) is one of the key enablers of small cell deployment, since the higher overall system efficiency is approximated by PA characterizations of Power Added Efficiency (PAE) and linearity. Consequently, the power efficient and ultrawideband PAs that enable multiple bands and carriers to be supported in one small cell are highly demanded. Different techniques have been proposed in the design of broadband amplifiers, usually in low noise applications, such as the Travelling wave amplifier [1], Lossy matched amplifier [2], Feedback amplifier [3], and amplifiers with resistive harmonic terminations [4]. The drawbacks of these approaches are the large circuit size, the low output power and low PAE performance. In order to enhance the efficiency, wideband switch-mode power amplifiers or harmonic tuning techniques, such as Class-E and Class-F, have drawn the most attention and many broadband amplifiers using waveform engineering have been reported in the literature [5-7]. However, these amplifiers show internal narrowband performance due to the device parasitics and require high-quality factor matching networks. Besides, Class-E PA strongly relies on the operating frequency to act as an ideal switch and at higher frequencies, 
the switching time may degrade its efficiency. Cripps in [8], proposed the Class-J PA solution for switching-mode amplifications, that opened possibilities into realizing bandwidth-efficiency PAs, by employing a capacitive harmonic load. Class-J enhances the voltage component by a purely reactive second harmonic, therefore, the linearity of the PA is not scarified by improving the efficiency. In [9], the harmonic-generation properties of the transistor's linear and nonlinear output capacitor are explored, and this work indicated that by using the purely resistive fundamental load impedance and a linear output capacitor the efficiency can be improved. In [10], a dual-band fundamental impedance matching for concurrent continuous Class-J PA is proposed and $68 \%$ drain efficiency at $1.8-2.8 \mathrm{GHz}$ has been achieved.

The main purpose of this paper is to represent the systematic approach of Class-J PA design in detail, that provides high-efficiency over a wide bandwidth. The design methodology includes the selection of a proper semiconductor technology, the appropriate Class-J operating condition (DC and RF), and source pull/load-pull simulations, according to the desired specifications and stability assessment. The major design requirements in this work include $10 \mathrm{~W}$ RF output power over 1.5 to $3.2 \mathrm{GHz}$, PAE greater than $50 \%$ and intermodulation distortion better than $-20 \mathrm{dBc}$. To ensure an optimal match, in the desired bandwidth, between the active device and the passive PA networks, wideband bias networks and wideband input/output matching networks are designed. Finally, as a result of these considerations, a broadband 10W GaN HEMT PA with more than one-octave bandwidth has been proposed.

The work is organized in the following sections. Section 2 introduces the Class-J theory of operation and optimal load impedances, at the fundamental and second harmonic terminations. The design topology and methodology of the Class-J PA is presented in Section 3. Also, in this section, the ADS simulation results will justify the efficient operation of the switch-mode PA by meeting the specifications. Finally, the main conclusions are discussed in section 4 .

\section{Operation characteristics of a Class-J power amplifier}

The key difference between the Class-J PA and other related PA modes is the requirement for a specific capacitive second harmonic termination that generates a rectified sine-wave. In this mode of operation, the combination of a reactive component at the fundamental and a low capacitance value can restore the RF power and efficiency. In such a way, the harmonics flowing into the capacitor generates the reactive voltage component that allows a higher fundamental component while the fundamental matching network blocks the higher harmonic currents. The drain voltage and current waveforms are characterized by half-sinusoidal waves with a phase shift between them and can be expressed by a Fourier series expansions in equations (2) and (3):

$$
\begin{gathered}
V_{d, B}(\omega t)=V_{d c}(1+\sin (\omega t)) \\
V_{d, J}(\omega t)=V_{d c}(1+\sin (\omega t))(1+\cos (\omega t)) \\
=V_{d c} *[1+\sin (\omega t)+\cos (\omega t)+1 / 2 \sin (2 \omega t)] \\
I_{d, J}(\omega t)=I_{\max }\left(\frac{1}{\pi}+\frac{1}{2} \sin (\omega t)-\frac{2}{3 \pi} \cos (2 \omega t)\right)
\end{gathered}
$$


Class-J drain voltage can be achieved by a shift of the Class-B PA drain voltage, given in equation (1). It is easy to see that the addition of the sine and cosine functions provides a phase shift for the component at the fundamental frequency and the second harmonic voltage contributes with a term that adds in phase to boost the fundamental. Therefore, the voltage waveform consists of DC, fundamental and second harmonic voltage components.

$$
\begin{gathered}
V_{d, J}(\omega t)=\left(V_{d c}-V_{k}\right)(1+\sin (\omega t))(1+\alpha \cos (\omega t)) \\
V_{d, J}(\omega t)=\left(V_{d c}-V_{k}\right)[1+\sin (\omega t)+\alpha \cos (\omega t)+0.5 \alpha \sin (2 \omega t)]
\end{gathered}
$$

By adding a factor to the front of the cosine function $(\alpha)$ in equation (5), which sweeps from -1 to 1 , the result is a family of voltage waveforms[11]. The Class-J is the case where $\alpha$ is equal to 1 , and class-B happens to be the case where $\alpha$ equals 0 , and there is also the mirror image of Class-J where the $\alpha$ equals -1 . Each voltage waveform, when combines with the baseline current waveform, generates the exact same overall RF power and efficiency as the baseline Class B PA. The importance of the Class-J design principle is to shift the phasing of the current and voltage waveforms, without changing their shape, in such a way, the second harmonic voltage and current components are in quadrature phase. The corresponding load impedance at the fundamental and second harmonics are as follows:

$$
\begin{gathered}
Z_{L, J @ f_{0}}=\frac{\left(V_{d c}-V_{k}\right)(1+j)}{\frac{I_{\max }}{2}}=\frac{2\left(V_{d c}-V_{k}\right)}{I_{\max }}(1+j) \\
Z_{L, J @ 2 f_{0}}=\frac{-0.5\left(V_{d c}-V_{k}\right) j}{\frac{2 I_{\max }}{3 \pi}}=-\left(\frac{3 \pi}{8}\right) \frac{2\left(V_{d c}-V_{k}\right)}{I_{\max }} j
\end{gathered}
$$

As can be seen in equation (7), the load impedance at the second harmonic leads directly to a shunt capacitance value. As a result, the continuous Class-J scheme offers a potentially wideband and high efficiency operation.

\section{A broadband Class-J PA design in GaN HEMT technology}

In this proposed Class-J PA design, a Gallium-Nitride (GaN) high electron-mobility transistor (HEMT) is selected due to its electrical properties of wide bandgap, high power density and high thermal conductivity. The biasing condition can be defined by considering the tradeoff among desired gain, efficiency, IMD and RF output power. Theoretically, a bias point close to the cut-off region can provide high-efficiency in broadband applications. In broadband amplifiers, the gain variation over the bandwidth causes an undesired phenomenon due to the transistor nonlinear capacitance that exhibits negative resistance in some frequency bands. As a result, the parametric oscillations [12] at a given level of input power may occur that need to be compensated by a gain equalizer. In this work, the Class-J PA is stabilized through a parallel RC circuit, within the input matching network, and a series resistor at the gate bias network of the active device to avoid oscillation. Generally, a linear stability analysis of the PA is based on the Rollet factor and the stability circles. Figure 1. (a) shows the plotted load stability circle and potentially unstable region inside the unit circle. Besides, Figure 1. (b) indicates that the 
stability factor $(\mathrm{k})$, that is greater than one across the band frequency of 1 to $4 \mathrm{GHz}$ to ensure the unconditional condition. In the case of the PA showing spurious oscillations in large-signal, other stability assessment methods should be considered.

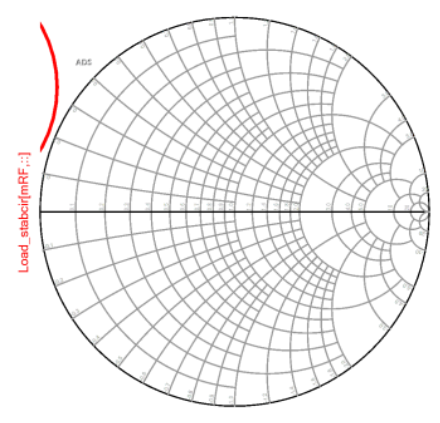

(a)

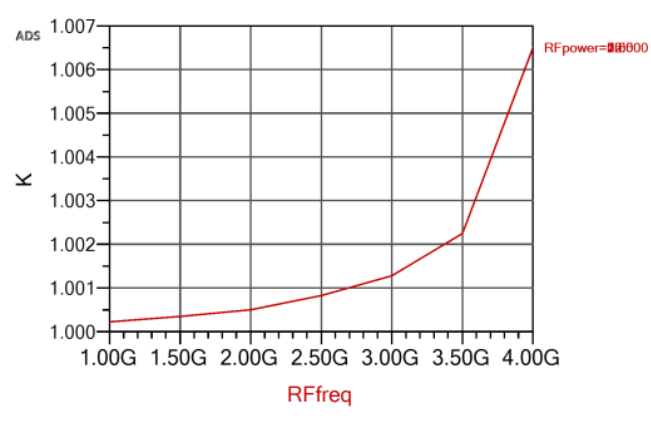

(b)

Fig. 1. (a) Load stability circle after adding stability network. (b) Stability factor.

\subsection{Network synthesis for a wideband bias tee}

For transforming the bias network into a wideband open circuit, a proper wideband bias line in a distributed network synthesis is needed. It is important to note that the characteristic impedance characterization of the transmission lines is set to the appropriate value, which could handle the current flowing through them. The maximum current that a microstrip line can carry as functon of the line width of $\omega$ is given by [13]:

$$
I=\omega \sqrt{A t \Delta T / \rho h}
$$

Where, $I$ is current at $\Delta T$ temperature above the one for the ground plane, $\rho$ is the resistivity of the conductor, $h$ and $t$ are the dielectric and strip thickness and $A$ is the thermal conductivity of the dielectric substrate. In this work, the minimum bias tee line width that can handle the maximum DC drain current of $1.9 \mathrm{~A}$ is calculated as corresponding to $80 \Omega$, for a Rogers $4350 \mathrm{~B}$ substrate. Therefore, the drain bias network is synthesized to include a set of decoupling capacitors, a radial stub and transmission lines to short out the RF signal from 1.5 to $3.2 \mathrm{GHz}$. Figure 2. illustrates the network synthesis for the desired drain bias tee. The capacitors with low value capacitance attenuate the RF signal at higher frequencies. In the center frequency, the $\lambda / 4$ line presents a high impedance for the RF signal path, which is inherently narrowband. In this regard, this line is tuned for higher frequency than the center of $2.4 \mathrm{GHz}$, to compensate the effects of a lower gain in the upper part of the frequency band. 


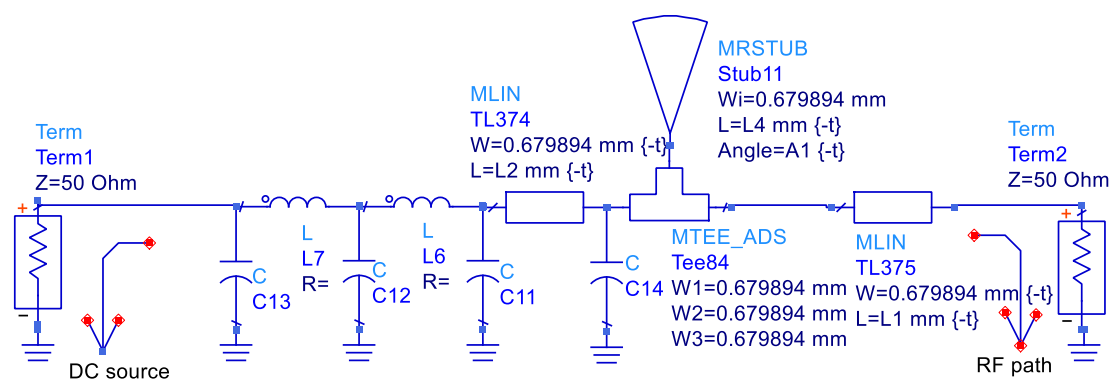

Fig. 2. Drain biasing network schematic.

On the other side, in the gate bias network, for reducing the gate current and noise from the power supply, the $\lambda / 4$ transmission line is replaced by an inductor and a resistor. Since a very small current may flow through the gate of the transistor, a resistor is sufficient for blocking the RF without introducing a voltage drop.

\subsection{Fundamental and Harmonic Matching Network Design}

The design procedure first identifies by the optimum load and source impedances. Loadpulling analysis is carried out at several frequencies between 1.5 to $3.2 \mathrm{GHz}$. The overlap between the efficiency contours that is bounded by at least 50\% PAE throughout the frequency band can provide the proper load impedance. Given the GaN HEMT device Cree CGH40010F, and based on closely spaced of impedance points, the level of degradation in PAE shows a less than $10 \%$, thus, the average optimum output impedance value around the center of the frequency band is selected. In the second step, the optimum second harmonic load that realized by device output capacitance must be determined at the frequency band. The second harmonic load termination can be defined by conducting the second harmonic load-pull simulation, while the device see the optimum fundamental load impedance and the higher harmonics are opencircuited. Figure 3.(a), shows the distribution of second harmonic terminations across the periphery of the Smith-chart. According to this figure, the extrinsic harmonic impedances of the Class $\mathrm{B}$ and $\mathbf{J}$ rotate in a continuous clockwise by almost the entire edge of the Smith chart. Passive components, by rotation on the Smith Chart can match only the reactance. Figure 3.(b), depicts the output loads predicted for the Class-J design space, where the load can be chosen from a family of impedances. Since the second harmonic is significantly expanded on the Smith chart compared to the fundamental impedance, the matching network for this harmonic termination is highly demanding for the specific frequency range. The Class-J amplifier is very sensitive to $2^{\text {nd }}$ harmonic termination that shows a substantial outcome on PA efficiency. 


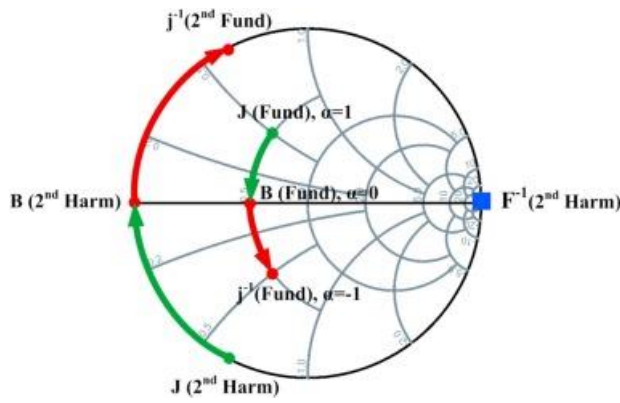

(a)

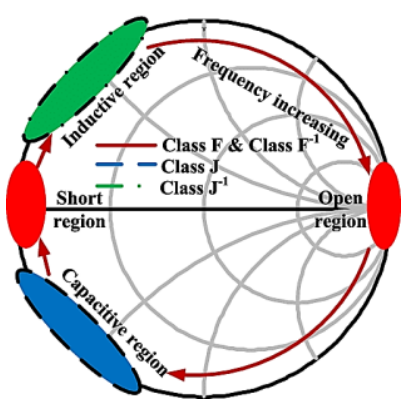

(b)

Fig. 3. Class-J design space and fundamental and second harmonic load impedances [14].

The next step is a systematic method to design wideband matching networks that transform the device complex impedances to the resistive load and source impedances of $50 \Omega$. However, the internal feedback and device parasitics, at higher frequencies, have a significant impact on the performance of the PA, where the input impedance is much smaller. For this purpose, the most promising topology to apply multiple stages for matching circuits [15]. This method can suppress the harmonics significantly and provides a wide frequency bandwidth with minimum power gain ripple. On the other hand, the narrowband behavior can be realized by an infinite number of matching sections. In this regard, the Chebyshev matching network [16], with a finite number of parts, is selected for the minimum reflection coefficient, as an approximation to the ideal network. Typically, for the low impedance values, the design methods of the Chebyshev multisection transformer is based on the transforming low-pass filters (LPF) of a ladder configuration. Figure 4. illustrates the LPF that consists of series inductances with shunt capacitances, with different transformation ratios $r=R_{1} / R_{2}$. Where $R_{1}$ is the characteristic impedance of the network and $R_{2}$ is the input resistance of the LPF. The output impedance is resistive because the capacitive reactance copupling that is compensated by the inductance. Moreover, the resistive matching circuit may also improve the amplifier stability and reduce its size and cost. Here, the ratio of the output filter is $50 / 17=2.9$. According to the Chebyshev low-pass Table [17], the required order for the output filter is $n=4$. The normalized admittance of $g$ element for the low pass matching network can be calculated as $g_{3}=r g_{2}$ and $g_{4}=g_{1} / r$, while $g_{1}$ and $g_{2}$ are given in [17] for different transformation ratios. The series section of inductance can be realized as a short circuited transmission line and the shunt capacitance can be replaced by open circuited shunt transmission lines. Values of the dc blocking capacitors are often chosen at picofarad due to their self-resonance frequency and low effective resistance at the fundamental frequency.

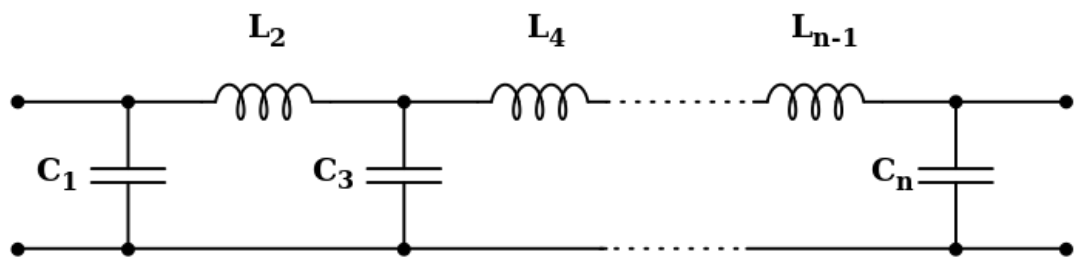

Fig. 4. Ladder topology of low-pass filter

$$
C_{1}=g_{1} / 2 \pi\left(f_{2}-f_{1}\right) R_{1}, C_{3}=g_{3} / 2 \pi\left(f_{2}-f_{1}\right) R_{1}
$$




$$
L_{2}=g_{2} R_{1} / 2 \pi\left(f_{2}-f_{1}\right), L_{4}=g_{4} R_{1} / 2 \pi\left(f_{2}-f_{1}\right)
$$

One of the most critical aspects of designing a broadband power amplifier lies in the inconsistent to achieve lower reflection coefficient over wide bandwidth. The frequency bandwidth with minimum reflection coefficient is defined by a loaded quality factor of $Q_{L}=$ $\omega_{0} \tau$ or $Q_{L}=1 / \omega_{0} \tau$ for series or parallel RC/RL circuits, where $\omega_{0}=\sqrt{\omega_{1} \omega_{2}}$ is the center bandwidth frequency between $\omega_{1}$ and $\omega_{2}$, and $\tau=R C=L / R$. In order to facilitate the wideband input matching network design, $Q_{L}=1$ is chosen in this work for the source impedance. Figure 5. show the applied network that satisfied the wideband criteria. The independent design of the fundamental and harmonic matching networks allows for the suppression of the harmonic bands and for providing an efficient and wideband amplification.

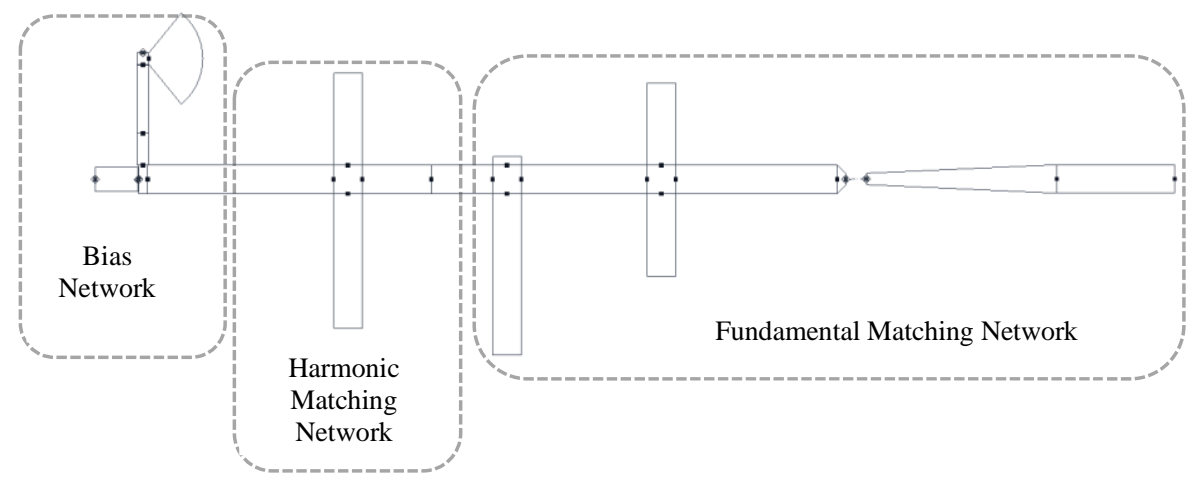

Fig. 5. Microstrip PCB layer of the load matching network.

The port matching is important to improve the gain over spectrum bandwidth. 1 . In such a case, the power gain at the lower frequencies will be sacrificed to reimburse gain roll-off. The initial approach for selecting the average optimum source impedance is to perform source-pull simulations over the band. Figure 6. demonstrates the frequency response of the input (a) and output(b) matching networks that are flat over the amplifier frequency band resulting in an flat transducer gain of the Class-J PA.

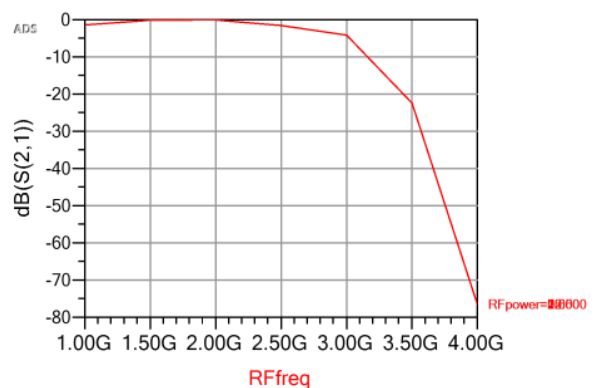

(a)

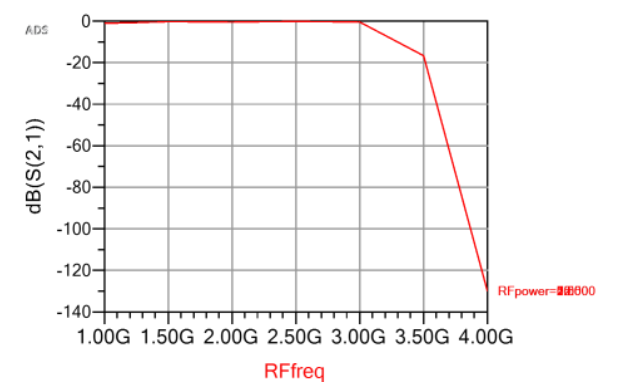

(b)

Fig. 6. S-parameter of the distributed elements of the matching networks. (a) IMN, (b) OMN

\subsection{Simulation Results}


The power amplifier is biased at $28 \mathrm{~V}$ drain voltage, $-3.1 \mathrm{~V}$ gate voltage and excited by a 10 $\mathrm{dBm}$ input RF power level over the 1.5 to $3.2 \mathrm{GHz}$. Simulation results in Figure 7. (a) indicate that a simulated peak drain efficiency of $70 \%$, at center frequency, together with the average PAE of $55 \%$ and an output RF power of $40 \mathrm{dBm}$ have been achieved. Since the optimum fundamental load and second harmonic are found from separate optimization, the optimum combination may not have been achieve, which would result in the degradation of the overall RF performance. The PA is swept over the 1.5 to $3.2 \mathrm{GHz}$ bandwidth, in $200 \mathrm{MHz}$ frequency steps, for a single tone. The transducer power gain in Figure 7. (b) shows the gain flatness between 10 to $13 \mathrm{~dB}$ within the entire bandwidth, which can be considered as a stable and satisfactory performance for the wideband PA.

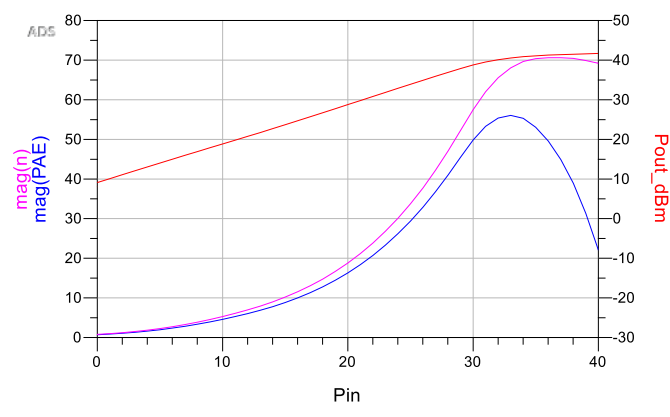

(a)

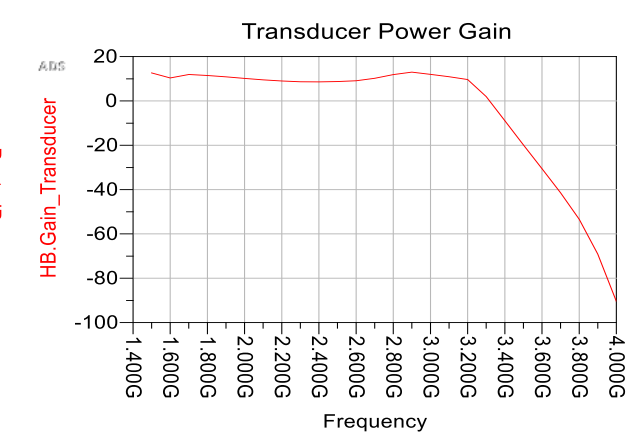

(b)

Fig. 7. (a)Simulated fundamental RF output power, power added efficiency and drain efficiency. (b) Simulated gain flatness vs frequency.

The simulated time-domain drain voltage and current waveforms are plotted in Figure 8. (a). Class-J uses a phase shift between the voltage and current waves to provide the purely reactive second harmonic termination, however, due to the phase overlap between the current and voltage waveforms, the performance of the Class-J mode is degraded. Drain waveforms of a Class-F PA are shown in Figure 8. (b) [18], the voltage waveform is shaped by summation of odd harmonics to approximate a square wave. Therefore, the harmonic shaping of the voltage waveform improves the fundamental voltage component for higher output power. Whereas, Class-J PA increases the fundamental voltage component through the second harmonic voltage as a capacitive load. The key to obtaining the maximum efficiency in Class-J PA lies in the reactive tuning of the fundamentals. In such a way, the second harmonic generates the reactive voltage component by flowing into the capacitor, which results in a higher fundamental component.
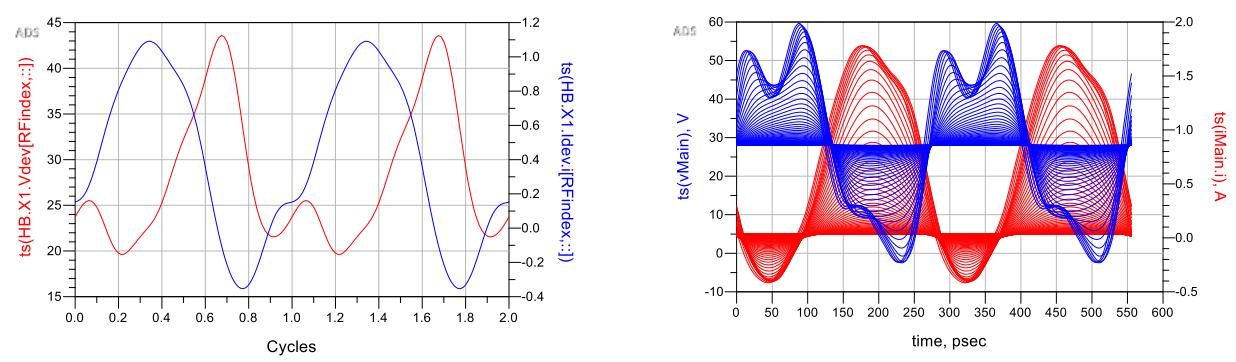
Fig. 8. Normalized current and voltage waveforms for (a) Class-J PA, (b) Class-F PA.

The IMD characteristic of the PA for two tones CW input signals with equal amplitude as shown in Figure 9. (a). The input powers are simultaneously swept from 10 to $30 \mathrm{dBm}$, and the IMD throughout the band is below $-21 \mathrm{dBc}$, which indicates satisfactory linearity performance. Figure 9. (b) shows the AM/PM phase characteristic of the designed J-PA at high power levels. The Voltage dependency of the capacitive parasitics in the device affects the linearity and general characteristic of the PA. However, the Class-J PA provides reasonable linearity as the Class-B mode due to the absence of a resonant impedance condition.

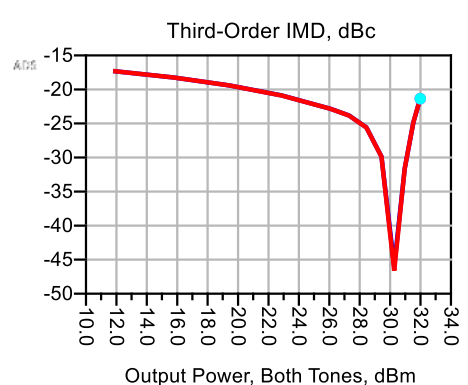

(a)

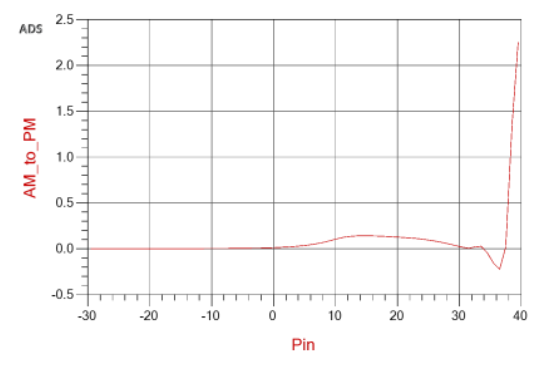

(b)

Fig. 9. Simulated IMD performance showing third-order IMD vs output power

The simulated performance of the designed J-PA compares with the measured performance of recently reported wideband Class-J amplifiers in Table 1., confirms that the presented wideband Class-J PA offers acceptable performance in terms of efficiency and gain for $5 \mathrm{G}$ wireless communication systems combining wideband and high efficiency.

Table 1. Summary of the wideband power amplifiers

\begin{tabular}{llll}
\hline Ref. & BW(GHz) & Gain $(\mathrm{dB})$ & Drain Eff \\
\hline$[19] 2009$ & $1.35-2.25(50 \%)$ & 11 & $60-70 \%$ \\
{$[20] 2018$} & $3.3-3.75$ & $11.8-13.5$ & $58 \%-71 \%$ \\
{$[21] 2011$} & $2.3-2.7(15 \%)$ & 11 & $60 \%$ \\
{$[22] 2017$} & $1.6-2.6$ & $15-16.3$ & $60-70 \%$ \\
This work & $1.5-3.6$ & $10-13$ & $58-70 \%$ \\
\hline
\end{tabular}

\section{Conclusion}

In this paper, a theory expressions of Class-J PA mode and a systematic design approach of a wideband operation and high efficient Class-J PA that meets the stringent requirements of 5G transmitters are described. Exploiting waveform engineering operation on the second harmonic loading, shows a remarkable improvement on efficiency. A broadband PA based on 
packaged GaN HEMT manufacturer's model over the frequency range of 1.5 to $3.2 \mathrm{GHz}$ is designed and simulated. In order to obtain a wideband behavior, the PA gain variation is controlled by a stabilizer that compensate the device parasitic capacitances. Multisection input and output matching networks are designed and their parameters optimized using formulas and special tables. The topology of the fundamental matching network is responsible for the enhancing the output device capacitance of device, which dominates the second harmonic impedance. Independent control of the harmonic circuit reduced the complexity of design procedure. The designed broadband Class-J PA can provide a maximum 55\% PAE and 10W output power and $72 \%$ fractional bandwidth.

Acknowledgments. This work is supported by the European Union's Horizon 2020 Research and Innovation program under grant agreement H2020-MSCA-ITN-2016-SECRET-722424.

\section{References}

[1] Robertson, I.D.: RFIC and MMIC Design and Technology. IEE Circuits, Devices and systems Series. UK (2001)

[2] Moazzam, M.: A high gain dual-fed single stage distributed amplifier, IEEE MTT-S International Microwave Symposium Digest. Vol. 3, pp. 1409-1412 (1994)

[3] Virdee, G.: Broadband Microwave Amplifiers, Artech House. Norwood. (2004)

[4] Andersson, M. Moon, J. Fager, C. Kim, B.: Decade bandwidth high efficiency GaN HEMT power amplifier designed with resistive harmonic loading, IEEE MTT-S International Microwave Symposium Digest. (2012)

[5] Raab, F.: Class-f power amplifiers with maximally flat waveforms, IEEE Trans. Microw. Theory Tech. Vol. 45, pp. 2007 -2012 (1997)

[6] Grebennikov, A and Sokal, N.: Switchmode RF Power Amplifiers, Newnes (2007)

[7] Maryam, S. Issa, E. Jonathan, R: A survey on RF and Microwave Doherty Power Amplifier for Mobile Handset Application, Electronics. Vol. 8, pp. 1-31 (2019)

[8] Cripps, S.C.: RF power amplifiers for wireless communications, Artech House. UK (2006)

[9] Junghwan, M. Jungjoon, K. And Bumman, K.: Investigation of a Class-J Power Amplifier With a Nonlinear Cout for Optimized Operation, IEEE Trans. On Microw. Theory Tech. Vol. 58, pp. 2800$2811(2010)$

[10] Zhenxing, Y. Mingyu Li, Yao, Y. Zhijiang, D. Tian, L. Yi, J.: Design of Concurrent Dual-Band Continuous Class-J Mode Doherty Power Amplifier with Precise Impedance Terminations. IEEE Microwave and Wireless Components Letters. Vol. 29, pp. 348-350 (2019)

[11] Steve. C. C, Paul. J. T, Alan. L. C, Jonathan. L, Johannes. B, : On the continuity of high efficiency modes in linear RF power amplifiers. IEEE Microw. Wireless Compon. Lett., vol. 19, no. 10, pp. 665667, (2009).

[12] Almudena, S. Sanggeun, J. And David, R.: Stability Analysis and Stablization of power amplifier, IEEE Microwave magazine. pp. 51-65 (2006)

[13] Nam-Tae, K.: Ultera-Wideband bias-tee design using distributed network synthesis, IEICE Electronics Express. Vol. 10, pp. 1-8 (2013)

[14] Samarth S, Karun, R. Patrick, R.: Continuous Class-B/J Power Amplifier Using Nonlinear Embedding Technique, IEEE Transactions on Circuits and Systems II. (2016)

[15] Nam-Tae, K.: Ultera-Wideband bias-tee design using distributed network synthesis, IEICE Electronics Express. Vol. 10, pp. 1-8 (2013)

[16] Andrei, G. Narendra, K. Binboga, S.: Broadband RF and Microwave Amplifiers. Taylor \& Francis Group, LLC. UK (2016)

[17] Seyed Alireza, M. Jianwei, A. Yueyun, Ch. Zhi, L.: Systematic Approach for Design of Broadband, High Efficiency, High Power RF Amplifiers, ETRI Journal.Vol. 39, pp. 51-61 (2017) 
[18] Maryam, S. Issa, E. Jonathan, R: Advancement of a Highly Efficient Class-F power Amplifier for 5G Doherty Architectures, IEEE 2nd 5G World Forum. pp. 86-90 (2019)

[19] Peter, W. Jonathan, L. Johannes, B. Paul, J. T. and Steve C. C.: Methodology for Realizing High Efficiency Class-J in a Linear and Broadband PA, IEEE Trans. On Microw. Theory Tech. Vol. 57, pp. 3196-3204 (2009)

[20] Chaoyi, H. Songbai, H. AND FEI, Y.: Design of Broadband Modified Class-J Doherty Power Amplifier with Specific Second Harmonic Terminations, IEEE Access. Vol. 6, pp. 2531-2540 (2018) [21] Neal, T. Anding, Z. Thomas, J.: Class-J RF Power Amplifier with Wideband Harmonic Suppression, IEEE Microwave, MTT-S International Symposium. USA (2011)

[22] Seyed Alireza, M. Fuhong, L. Xianwei, Zh. Sani, A.: Design of broadband, high-efficiency and high-linearity GaN HEMT Class-J RF power amplifier, High Power RF Amplifiers, Progress in Electromagnetics Research C.Vol. 72, pp. 177-186 (2017) 